\title{
Prebiotic efficiency of blue green algae on probiotics microorganisms
}

\begin{abstract}
Blue green algae also called cyanobacteria are considered to be the photosynthetic prokaryotes. Their similarities with both higher plants and simple bacteria have made them ideal model systems for intensive research in varied spheres of biology. Their role in nitrogen fixation is well known but their role as prebiotics to support the growth of probiotic bacteria is an upcoming field of study. Prebiotics includes indigestible carbohydrates mainly oligosaccharides that benefit the host organism by selectively stimulating the growth or activity of one or a limited number of probiotic bacteria in the colon. Spirulina, a cyanobacterium is a photoautotrophic microorganism, widely distributed in nature and is consumed as human food supplement for centuries because of its best known nutritional value. It contains $78 \%$ proteins, vitamins, $4-7 \%$ lipids, minerals, carbohydrates and some natural pigments. Due to the presence of these phytonutrients, it has corrective properties against several diseases like cancer, hypertension, hypercholesterolemia, diabetes, anaemia etc. Research studies have reported that the extracellular products produced by Spirulina platensis significantly promotes the growth of lactic acid bacteria such as Lactococcus lactis, Streptococcus thermophilus, Lactobacillus casei, Lactobacillus acidophilus, and Lactobacillus bulgaricus. The present paper would focus on the prebiotic efficiency of certain blue green algae on probiotic microorganisms.
\end{abstract}

Keywords: prebiotic, probiotic bacteria, blue-green algae, phytonutrients
Volume 4 Issue 4 - 2017

\author{
Sneh Gupta, ${ }^{2}$ Charu Gupta,' Amar P Garg, ${ }^{3}$ \\ Dhan Prakash' \\ 'Amity Institute for Herbal Research \& Studies, Amity University \\ UP, India \\ ${ }^{2}$ Department of Zoology, RG (PG) College, India \\ ${ }^{3}$ Department of Microbiology, CCS University, India
}

Correspondence: Charu Gupta, Amity Institute for Herbal Research \& Studies, Amity University UP, Sector-125, Noida-20I3।3, India, Email charumicro@gmail.com

Received: February 21, 2017 | Published: April 6, 2017

\section{Introduction}

According to definition, probiotics are defined as selected, viable, microbial dietary supplements that when introduced in sufficient quantities, beneficially affect human organism through their effects in the intestinal tract. ${ }^{1,2}$ There are large number of probiotics currently available in fermented dairy products like yoghurt, cheese, curd, icecream etc. All these products contain diverse group of microorganisms preferably belonging to lactic acid bacteria. Some of them are natural inhabitants of the intestinal tract and others as fermentative lactic acid bacteria used in the food industry for imparting improved flavor, texture and processing and preservative properties. Some species are administered to humans as live microbial supplements, which positively influence our health mainly by improving the composition of intestinal microbiota. For this reason, they are called probiotics.

Some selected strains of Lactobacillus, Bifidobacterium, Streptococcus, Lactococcus and Saccharomyces have been promoted in food products because of their reputed health benefits. ${ }^{3}$ The probiotic effect of these microorganisms include prevention of constipation in elderly people, preventing diarrhea, stimulation of immune system, ${ }^{4}$ lactose intolerance, reduction in cholesterol levels in blood and prevention of cancer. ${ }^{5}$ Apart from these therapeutic benefits, probiotics also offer protection against many opportunistic human pathogens. ${ }^{6,7}$ Ingestion of probiotic bacteria may actually improve many of the host's physiological processes. These include a reduction in mutagenic enzymes such as $\beta$-glucuronidase, nitroreductase, and choloylglycine hydrolase. ${ }^{8}$

To observe a positive health effect of their consumption, a minimum level of live microorganisms is required. This level, depend upon the strains used and the required health effect ranges between 108 and $1011 \mathrm{CFU} / \mathrm{gl}$. It has corrective properties against several diseases like cancer. ${ }^{9}$ Prebiotics are the foods for probiotic bacteria. They are defined as non-digestible or low-digestible food ingredients that benefit the host organism by selectively stimulating the growth or activity of one or a limited number of probiotic bacteria in the colon. ${ }^{10}$ This role is played by fermentable carbohydrates, which are not digested or poorly digested in the small intestine and stimulate, preferentially, the growth of bifidobacteria and some Gram-positive bacteria, belonging to the probiotic bacteria administered to humans. Complex carbohydrates pass through the small intestine to the lower gut where they become available for some colonic bacteria but are not utilized by the majority of the bacteria present in the colon. Lactulose, galacto-oligosaccharides, fructo-oligosaccharides, inulin and its hydrolysates, malto-oligosaccharides, and resistant starch are prebiotics commonly used in human nutrition. The main end products of carbohydrate metabolism are short-chained fatty acids, namely acetate, butyrate and propionate, which are further used by the host organism as an energy source.

The most common oligosaccharides are inulin and its hydrolysates and oligofructans. They can be found in chicory, topinambur, onion, garlic, asparagus, artichoke, leek, bananas, tomatoes and many other plants. Prebiotic oligosaccharides can be produced in three different ways: by extraction from plant materials, microbiological synthesis or enzymatic synthesis, and enzymatic hydrolysis of polysaccharides. ${ }^{11}$ In practice, combined mixtures of probiotics and prebiotics are often used because their synergic effects are conferred onto food products. For this reason, such mixtures are called synbiotics.

The production of prebiotics at industrial scale faces several challenges, including the use of novel techniques and economical sources, and the low-cost production. Most oligosaccharides with prebiotic status are normally obtained by enzymatic treatment of cheap raw materials such as sucrose, lactose and plant derivatives. ${ }^{12}$ The amount and nature of oligosaccharides formed depend upon several factors such as the enzyme source, the concentration and nature of the substrate and the reaction conditions. Nevertheless, the current 
processes used to obtain oligosaccharides have very low yields, thus increasing the production cost. ${ }^{13}$ Therefore, prebiotics from marine and freshwater algae are an attractive alternative source for promoting the growth of Lactobacillus and Bifidobacterium spp. which are the widely studied probiotic strains of lactic acid bacteria.

\section{Cyanobacteria as prebiotics}

Macroalgae are regarded as a rich source of sulfated polysaccharides, and the particular types of polysaccharides varied depending on the taxonomic group. The key function of these relatively high molecular weight polysaccharides is that they are rich in hydroxyl $(\mathrm{OH})$ groups, making them hydrophilic. They are known to establish intra-chain $\mathrm{H}$-bond networks, making them stiff, rigid and suitable as thickeners. The regularity of their structures also promotes their interaction with external ions and inter-chain H-bonding (e.g. gelation).${ }^{14}$ Different carbohydrates including ulvans, carrageenan, or alginates are extracted from macroalgae and are widely used in the food and pharmaceutical industries as functional ingredients.

Spirulina, a cyanobacterium is a photoautotrophic microorganism, widely distributed in nature and is consumed as human food supplement for centuries because of its best known nutritional value. It contains $78 \%$ proteins, vitamins, $4-7 \%$ lipids, minerals, carbohydrates and some natural pigments. Due to the presence of these phytonutrients, it has corrective properties against several diseases like cancer, hypertension, hypercholesterolemia, diabetes, anemia etc. Recently Parada ${ }^{15}$ reported the growth promotion effect of lactic acid bacteria by $S$. platensis.

Spirulina has been used for many years as human food because of its high protein content and nutritional value. Some strains also produce bioactive substances that may inhibit or promote microbial growth. Lactococcus lactis, Streptococcus thermophilus, Lactobacillus casei, Lactobacillus acidophilus, and Lactobacillus bulgaricus were grown in rich media, de Man Rogosa Sharpe media (MRS), as well as in minimal saline medium in the presence and absence of extracellular products obtained from a late log phase culture of Spirulina platensis in Zarrouk medium. In both MRS and RM media, the extracellular products significantly promote the growth of the lactic acid bacteria assayed. $^{15}$

Laminarans (or laminarins), found especially in Laminaria/ Saccharina, were recognized as dietary fiber with prebiotic properties, with applications as substrate for probiotic bacteria. ${ }^{16}$ Furthermore, laminaran seems to play a role in regulating the gut metabolism by interfering with gut goblet cells of the epithelial layer and stimulates the release of mucus. Marine polysaccharides present an enormous variety of structures which are still exploited and should therefore be considered as an extraordinary source of prebiotics. ${ }^{17}$ Polysaccharides of algal origin such as alginates, laminarins, fucans and their derivatives have prebiotic potential that have been used for decades to enhance animal and human health ${ }^{14}$

\section{Algal Species with Prebiotic Potential}

There are a number of algal species that have been used in the food and pharmacological industries. The microscopic descriptions of these algal species are presented along with prebiotic properties.

\section{Spirulina platensis}

Spirulina platensis(S. platensis) is a planktonic, filamentous blue- green algae found in many freshwater environments including ponds, lakes, and rivers. Spirulina platensis contains about $13.6 \%$ carbohydrates; some of which are glucose, rhamnose, mannose, xylose and galactose. Spirulina platensis biomass is known to stimulate bacterial growth, and studies performed indicated that the algae consumes nitrogen from the growth medium and releases extracellular carbohydrates and other growth substances which, may be responsible for stimulating growth of Lactobacilli (and other lactic acid-producing strains). ${ }^{15}$

\section{Chlorella species}

Chlorella spp. is unicellular green algae of Chlorophyta found in both fresh and marine water. Polysaccharide complexes from Chlorella pyrenoidosa and possibly, Chlorella ellipsoidea, contain glucose in combination with any of the following sugars like galactose, rhamnose, mannose, arabinose, $\mathrm{N}$-acetylglucosamide and $\mathrm{N}$-acetylgalactosamine. ${ }^{18}$ An acidic polysaccharide was isolated from Chlorella pyrenoidosa containing mostly rhamnose (52\%) with both arabinose and galactose in about equal amounts (12 and $13 \%$, respectively). These complexes are believed to have immunostimulating properties, inhibiting the proliferation of Listeria monocytogenes and Candida albicans. ${ }^{19,20}$

\section{Tetraselmis species}

Tetraselmis is a sizeable genus (more than 50 species) of green flagellates. Most species are known from inshore marine environments, tide pools in particular, but a few freshwater species are also known. ${ }^{21}$ The cell walls (theca) of Tetraselmis spp. consist mainly of acidic polysaccharides ( $82 \%$ of dry weight). ${ }^{22}$

\section{Dunaliella salina}

Dunaliella salina (D. salina) is a unique unicellular species of Chlorophyta with no cell wall found in saline environments. ${ }^{23}$ This alga has been reported to produce extracellular polysaccharides that are known to have immuno-stimulant, anti-viral and anti-tumor activities. $^{24}$

\section{Scenedesmus species}

Scenedesmus spp. are ubiquitous organisms and are frequently dominant in freshwater lakes and rivers. They are well-known for their nutritional value and considered as potential food additives. ${ }^{25}$ Scenedesmus spp. has also been used for producing lipid and carbohydrate suitable for making liquid biofuels. ${ }^{26}$ Colony formation in algae is linked to polysaccharide production however polysaccharide from these algae is limited. ${ }^{27}$

\section{Chlorococcum species}

The marine green alga Chlorococcum is a unicellular spherical strain with cells of diameter about $10 \mu \mathrm{m}$ belonging to Chlorococcaceae family. ${ }^{28}$ It is of interest due its tolerance to high $\mathrm{CO} 2$ concentrations and the fact that it can grow to high cell density. ${ }^{29}$ Just like all other microalgae, it can produce value added by-products that can enable their processes more economical. Some of the examples include their use in medicinal and pharmaceutical industry as well as health drinks for their immuno-stimulatory, antioxidant, antiviral, and anticancer activities. ${ }^{30}$

\section{Cylindrospermum species}

Cylindrospermum usually inhabits soft, acidic freshwater lakes and is one of the filamentous, heterocystous and non-branched cyanobacteria, classified traditionally in Nostocaceae family. The filaments form fine or compact benthic mats or colonies and may be 
epiphytic or metaphytic. ${ }^{31}$ Cylindrospermum spp are employed in agriculture as biofertilizers and soil conditioners. They are capable of fixing atmospheric nitrogen and are effectively used as biofertilizers its application is useful for 39 the reclamation of soils. ${ }^{32}$

\section{Synbiotic Effect of Algal Oligosaccharides}

A combination of probiotic(s) and prebiotic(s) constitutes a synbiotic, which can stimulate and increase the survival of probiotic and autochthonous-specific strains in the intestinal tract. ${ }^{33}$ The combination of a prebiotic of plant material for example; inulin and a probiotic in one product has shown to confer benefits beyond those of either prebiotic or probiotic. ${ }^{2}$ Probiotic and prebiotic treatment has been shown to be a promising therapy to maintain and repair the intestinal environment. Consumption of healthy live microorganisms (lactic acid bacteria) with prebiotics such as inulin, galactooligosaccharide and oligo-fructose to mention a few, may enhance healthy colonic microbiota composition. This combination might improve the survival of the bacteria crossing the upper part of the GIT, thereby boosting their effects in the large bowel. ${ }^{34}$

The various research studies have shown that aqueous algal extracts from S. platensis, Chlorococcum, D. salina, S. magnus, Chlorella algal extracts are potential sources for prebiotic production. Spirulina platensis extract was regarded as the best algal source for prebiotic as it had a greater stimulatory effect on the growth of all three probiotic bacteria (L. lactis, B. longum and L. bulgaricus). Xylose and galactose characterized by HPLC in algal extracts make up oligosaccharides that function as prebiotic compounds for stimulation of probiotic bacteria. ${ }^{33}$ Thus there is a great scope for successful production of prebiotics from algal sources.

\section{Acknowledgements}

Authors are grateful to Dr. Ashok K. Chauhan, Founder President and Mr. Atul Chauhan, Chancellor, Amity University UP, Noida, India for the encouragement, research facilities and financial support.

\section{Conflicts of Interest}

The authors declare no conflict of interest.

\section{Funding}

None.

\section{References}

1. Zubillaga M, Weil R, Postaire E, Goldman C, et al. Effect of probiotics and functional foods and their use in different diseases. Nutrition Research. 2001;21(3):569-579.

2. Holzapfel WH, Schillinger U. Introduction to pre and probiotics. Food Research International. 2002;35: 109-116.

3. Puupponen Pimia R, Aura AM, Oksman Caldentey KM, et al. Development of functional ingredients for gut heath. Trends in Food Science \& Technology. 2002;13(1): 3-11.

4. Schiffrin EJ, Rochat F, Link Amster $\mathrm{H}$, et al. Immunomodulation in human blood cells following the ingestion of lactic acid bacteria. $J$ of Dairy Sci. 1995;78(3):491-497.

5. Lee NK, Park JS, Park E, et al. Adherence anti-carcinogenic effects of Bacillus polyfermenticus SCD in the large intestine. Lett Appl Microbiol. 2007;44(3):274-278.

6. Collado MC, Jalonen L, Meriluoto J, et al. Protection mechanism of probiotic combination against human pathogens: in vitro adhesion to human intestinal mucus. Asia Pac J Clin Nutr. 2006;15(4):570- 575.
7. Brook L. The role of bacterial interference in otitis, sinusitis and tonsillitis. Otolaryngol Head Neck Surg. 2005;133(1): 139-146.

8. Roos N, Katan MB. Effects of probiotic bacteria on diarrhea, lipid metabolism, and carcinogenesis: a review of papers published between 1988 and 1998. Am J Clin Nutr. 2000;71(2):405- 411.

9. Vanderhoof JA, Young RJ. Use of probiotics in childhood gastrointestinal disorders. J PediatrGastroenterol Nutr. 1998;27(3):323-332.

10. Manning TS, Gibson GR. Prebiotics. Best Pract Res Clin Gastroenterol. 2004;18(2):287-298.

11. Gulewicz P, Ciesiolka D, Frias J, et al. Simple method of isolation and purification of T-galactosides from legumes. J Agric Food Chem. 2014;48(8):3120-3123.

12. Figueroa González I, Quijano G, Ramirez G, et al. Probiotics and prebiotics: Perspectives and challenges. J Sci Food Agric. 2011;91(8):1341-1348.

13. Panesar PS, Panesar R, Singh RS, et al. Microbial production, immobilization and applications of $\beta$-D-galactosidase. Journal of Chemical Technology and Biotechnology. 2006;81(4):530-543.

14. O Sullivan L, Murphy B, Mcloughlin P, et al. Prebiotics from marine macro-algae for human and animal health applications. Mar Drugs. 2010;8(7):2038-2064.

15. Parada JL, Zulpa De Caire G, Zaccaro De Mulé MAC, et al. Lactic acid bacteria growth promoters from Spirulina platensis. Int $J$ Food Microbiol. 1998:45(3):225-228.

16. Deville C, Damas J, Forget J, et al. Laminarin in the dietary fiber concept. Journal of Science of Food and Agriculture. 2004;84(9):10301038 .

17. Senni K, Pereira J, Gueniche F, et al. Marine polysaccharides: a source of bioactive molecules for cell therapy and tissue engineering. Mar Drugs. 2011;9(9):1664-1681.

18. Lordan S, Ross RP, Stanton C. Marine bioactives as functional food ingredients: potential to reduce the incidence of chronic diseases. Mar Drugs. 2011;9(6):1056-1100.

19. Mata TM, Martins AA, Caetano NS. Microalgae for biodiesel production and other applications: a review. Renewable and Sustainable Energy Reviews. 2010;14(1):217-232.

20. Suárez ER, Kralovec JA, Noseda MD, et al. Isolation, characterization and structural determination of a unique type of arabinogalactan from an immunostimulatory extract of Chlorella pyrenoidosa. Carbohydrate Research. 2005;340(8):1489-1498.

21. Mohammadi M, Kazeroni N, Aboli MJ, et al. Fatty acid composition of the marine micro alga Tetraselmis chuii Butcher in response to culture conditions. Journal of Algal Biomass Utilazation. 2015;6(2): 49-55.

22. Becker B, Melkonian M, Kamerling JP. The cell wall (theca) of Tetraselmis striata (Chlorophyta): Macromolecular composition and structural elements of the complex polysaccharides. Journal of Phycology. 1998;34(5): 779-787.

23. Abu Rezq TS, Al Hooti S, Jacob DA. Optimum culture conditions required for the locally isolated Dunaliella salina. Journal of Algal Biomass Utilization. 2010;1(2):12-19.

24. Mishra A, Jha B. Isolation and characterization of extracellular polymeric substances from micro-algae Dunaliella salina under salt stress. Bioresource Technology. 2009;100(13):3382-3386.

25. Kumar SA, Magnusson M, Ward LC, et al. A green algae mixture of Scenedesmus and Schroederiella attenuates obesity-linked metabolic syndrome in rats. Nutrients. 2015;7(4):2771-2787. 
26. Ho SH, Chen WM, Chang JS (2010) Scenedesmus obliquus CNW-N as a potential candidate for $\mathrm{CO} 2$ mitigation and biodiesel production. Bioresour Technol. 2010;101(22):8725-8730.

27. Liu Y, Wang W, Zang M, et al. PSII-efficiency, polysaccharide production, and phenotypic plasticity of Scenedesmus obliquus in response to changes in metabolic carbon flux. Biochemical Systematics and Ecology. 2010;38(3):292-299.

28. Berberoglu H, Gomez PS, Pilon L. Radiation characteristics of Botryococcus braunii, Chlorococcum littorale, and Chlorella sp. used for CO2 fixation and biofuel production. Journal of Quantitative Spectroscopy and Radiative Transfer. 2009;110: 1879-1893.

29. Hu Q, Kurano N, Kawachi M, et al. Ultrahigh-celldensity culture of a marine green alga Chlorococcum littorale in a flat-plate photobioreactor. Appl Microbiol Biotechnol. 1998;49(6):655-662.
30. Skjånes K, Lindblad P, Muller J. BioCO2: A multidisciplinary, biological approach using solar energy to capture $\mathrm{CO} 2$ while producing $\mathrm{H} 2$ and high value products. Biomol Eng. 2007;24(4):405-413.

31. Hijova E, Chmelarova A. Short chain fatty acids and colonic health. Bratisl Lek Listy. 2007;108(8):354-358.

32. Harborne JB. Phytochemical methods: A guide to modern techniques of plant analysis. Chapman, London, 1973. p. 302.

33. Gourbeyre P, Denery S, Bodinier M. Probiotics, prebiotics, and synbiotics: impact on the gut immune system and allergic reactions. $J$ Leukoc Biol. 2011;89(5):685-695.

34. Jain M, Gupta K, Jain P, et al. Significance of probiotics and prebiotics in health and nutrition. Malaya Journal of Biosciences 2014;1(3):181-195. 